\title{
Interkulturelle Zusammenarbeit im Rahmen von Bildung für nachhaltige Entwicklung. Österreichische und indische Jugendliche forschen gemeinsam zum Thema intragenerationale Gerechtigkeit.
}

\author{
" martin.duer@ph-vorarlberg.ac.at, Pädagogische Hochschule Vorarlberg, BRG und BORG Feldkirch \\ ** lars.keller@uibk.ac.at, Institut für Geographie, Universität Innsbruck \\ ***anna.oberrauch@uibk.ac.at, Institut für Geographie, Universität Innsbruck
}

eingereicht am: 08.10.2015, akzeptiert am: 24.12.2015

\begin{abstract}
Dieser Beitrag beschreibt die theoretischen Hintergründe, den Ablauf und die Zielsetzung des Forschungsprojekts „AustrIndia-4QOL“. Schüler/innen einer 7. Klasse AHS aus Vorarlberg arbeiten über digitale Medien mit Gleichaltrigen aus einer Schule in Nordindien zusammen und setzen sich gemeinsam mit Fragen zur Schaffung hoher Lebensqualität unter dem Gesichtspunkt intragenerationaler Gerechtigkeit auseinander. Durch moderat konstruktivistisch gestaltete Lernsettings wird versucht, bei den Jugendlichen das Bewusstsein und die Handlungsbereitschaft für eine nachhaltige Lebensweise zu stärken.
\end{abstract}

Keywords: Bildung für nachhaltige Entwicklung, Globales Lernen, intragenerationale Gerechtigkeit, Lebensqualitätsforschung

Intercultural cooperation in education for sustainable development. Joint research by Austrian and Indian pupils into intragenerational justice

This paper describes the theoretical background, the process and aims of the research project AustrIndia-4QOL. With the help of digital media, students in the $11^{\text {th }}$ grade of an Austrian high school cooperate with same-age students of a school in Northern India. They deal with questions of how to create a high quality of life while accommodating intragenerational justice. Based on moderately constructivist learning designs, the materials aim to strengthen awareness of a sustainable lifestyle in young people and to encourage their willingness to take action.

Keywords: education for sustainable development, global learning, intragenerational justice, quality of life research

\section{$1 \quad$ Einleitung}

Die globalen Herausforderungen des 21. Jh. bedingen ein neues Verständnis von Entwicklung, das nicht mehr vom Fortschritts- und Wachstumsparadigma des Industriezeitalters ausgeht, sondern Aspekte der weltweiten Armutsbekämpfung, der globalen Migration oder der nachhaltigen Nutzung der natürlichen Ressourcen in den Mittelpunkt der Überlegungen stellt (Obrecht 2014). Überlegungen, die auch verstärkt in den Unterricht im Fach Geographie und Wirtschaftskunde (GWK) einfließen sollten. Im vorliegenden Forschungsprojekt mit dem Kurztitel „AustrIndia4QOL" (Austria and India for Quality of Life) wird versucht, der Frage nachzugehen, inwiefern es durch länderübergreifende Zusammenarbeit im GWK-Unterricht gelingen kann, bei Jugendlichen erhöhtes Bewusstsein und erhöhte Handlungsbereitschaft für eine nachhaltige Lebensweise zu erreichen. Dabei steht verstärkt der Aspekt der intragenerationalen Gerechtigkeit im Fokus, um eine hohe Lebensqualität für alle Menschen auf der Erde zu erreichen.

In diesem Sinne folgt das Projekt „AustrIndia4QOL“ den Zielen einer „Bildung für nachhaltige Entwicklung“ (BNE) der Vereinten Nationen. BNE zielt darauf ab, jedem Mensch die Möglichkeit zu bieten, sich Wissen, Kompetenzen, Einstellungen und Werthaltungen für die Gestaltung einer nachhaltigen Zukunft anzueignen (UNESCO 2015a). Moderner Unterricht setzt sich dementsprechend ebenfalls zum 
Ziel, Jugendliche zu befähigen, für die Zukunft die wesentlichen persönlichen und gesellschaftspolitischen Weichenstellungen reflektiert vorzunehmen. Die $\mathrm{Zu}-$ kunft der Menschheit entscheidet sich laut Schmidtke in diskursiven Prozessen, da die großen Herausforderungen nicht mehr mit einer als einzig richtig empfundenen Theorie gelöst werden können. Die komplexe(n) Wirklichkeit(en) erfordert/erfordern einen partizipativen Ansatz, der das "Sowohl-als-auch“ anstelle des „Entweder-oder“ in den Mittelpunkt stellt (Schmidtke 2009, 147). Für Jugendliche, als die Entscheidungsträger/innen von morgen, ist es daher essentiell, in authentischen Situationen zu lernen, sich Wissen und Meinungen zu erarbeiten und auszutauschen, auf partizipative Weise Kompromisse auszuhandeln, Lösungen zu entwickeln und begründete Entscheidungen zu treffen. Dies stärkt sie in ihrer Bereitschaft, nicht nur Verantwortung für ihr eigenes Leben, sondern auch Verantwortung für Menschen in anderen Regionen der Welt zu übernehmen (Kyburz-Graber 2010). Die heutigen Jugendlichen werden einerseits über Erfolg oder Misserfolg von Nachhaltigkeitsstrategien entscheiden und andererseits von den Folgen dieser Entwicklungen direkt und intensiv betroffen sein.

Im Folgenden werden zunächst theoretische Hintergründe des Forschungsprojekts „AustrIndia-4QOL“ erläutert und Projekte vorgestellt, deren Erkenntnisse in die Konzeption einfließen. Anschließend wird auf die Zielsetzungen, die konkrete Vorgangsweise sowie auf die Erwartungen an dieses Projekt eingegangen.

\section{Zentrale Aspekte des geplanten For- schungsprojekts}

Das heutige Konzept der nachhaltigen Entwicklung geht auf den 1987 von der sogenannten BrundtlandKommission veröffentlichten Bericht Our Common Future zurück (WCED 1987). Auf der United Nations Conference on Environment and Development 1992 in Rio de Janeiro wurde von 178 Nationen das Leitbild der nachhaltigen Entwicklung als globales Konzept für das 21. Jh. beschlossen - die sogenannte Agenda 21. Hauff (2012) betont vor allem zwei wesentliche Anforderungen dieses Leitbildes: Die gleichrangige und integrative Berücksichtigung von ökologischen, ökonomischen und sozialen Dimensionen und die Realisierung von intra- und intergenerationaler Gerechtigkeit. „Nur die Gleichrangigkeit und Zusammenführung der drei Dimensionen kann zu einer Sicherung bzw. zur Verbesserung der Lebensqualität der Menschen führen" (Hauff 2012, 7). Damit fand auf dieser Konferenz zum ersten Mal eine breite Diskussion über die enge Verbindung von Entwicklung und Umwelt statt (Schuler \& Kanwischer 2013). 2002 wurden auf der Konferenz in Johannesburg zusätzlich die Aspekte Menschenrechte und Grundfreiheiten, Frieden und kulturelle Vielfalt verstärkt betont. Besonders von Vertreterinnen und Vertretern des Globalen Lernens werden deshalb die ursprünglichen drei Dimensionen von Nachhaltigkeit (ökologisch, ökonomisch und sozial) um eine politische Dimension ergänzt. Diese vier Zieldimensionen sind zusätzlich immer unterschiedlichen kulturellen Interpretationen unterworfen (Schreiber et al. 2010). Die Bedeutung der kulturellen Dimension für die Umsetzung des Prinzips der Nachhaltigkeit nehmen Autoren wie z. B. Krainer und Trattnigg (2007) oder Heintel (2008) zum Anlass, unter dem Begriff der „kulturellen Nachhaltigkeit" eben diese kulturelle Komponente verstärkt einzufordern. Nur bei einer Berücksichtigung kulturell unterschiedlicher Strategien und Techniken kann aus Wissen auch eine Bereitschaft zum Handeln entstehen (z.B. Krainer \& Trattnigg 2007). Dazu müssen die Vertreter/innen der Industriestaaten im Sinne eines partizipativen Ansatzes lernen, mit den Vertretern der Entwicklungs- und Schwellenländern auf Augenhöhe zusammenzuarbeiten und die in anderen Teilen der Welt existierenden Problemlösungskompetenzen zu berücksichtigen (Obrecht 2014). „Tragfähige Lösungen können nicht einfach diktiert, sondern müssen unter möglichst vielen Beteiligten ausgehandelt werden" (Nagel 2010, 10). Heintel (2008, 116 ff.) fordert zudem eine neue „Kultur des Immateriellen", die sich gesellschaftlich durchsetzen müsse. Kulturelle Nachhaltigkeit steht dabei nicht als vierte Säule neben Ökologie, Ökonomie und Sozialem, da die jeweilige Kultur das Handeln in allen drei Bereichen durchdringt und bestimmt (Heintel 2008). Sowohl beim politischen als auch kulturellen Aspekt gehe es eher um die Art der Umsetzung, weshalb diese Aspekte im Sinne einer integrierenden oder querliegenden Dimension dargestellt werden (Oehme 2007).

\subsection{Bildung für nachhaltige Entwicklung (BNE) als Ausgangspunkt}

Der 2002 in Johannesburg abgehaltene „Weltgipfel für nachhaltige Entwicklung" bildete für die Generalversammlung der Vereinten Nationen den Anlass, den Zeitraum von 2005 bis 2014 zur „Dekade der Bildung für nachhaltige Entwicklung" zu erklären. Damit wird bezweckt, die Prinzipien der nachhaltigen Entwicklung in den nationalen Bildungssystemen zu verankern (Schuler \& Kanwischer 2013). Irina Bokova, Generaldirektorin der UNESCO, fordert im Vorwort des Education Report 2015, dass in einer sich ändernden Welt auch Bildung einen Wandel erleben muss. Nur mit neuen Formen von Bildung könnten jene Kompetenzen vermittelt werden, die die sich 
Tab. 1: Teilkompetenzen der Gestaltungskompetenz und deren Zuordnung zu den Kompetenzkategorien der OECD

(Quelle: De Haan 2008, 32)

\begin{tabular}{ll}
\hline Kompetenzkategorien der OECD & Teilkompetenzen der Gestaltungskompetenz \\
\hline Interaktive Verwendung von Medien und Tools & - Weltoffen und neue Perspektiven integrierend Wissen aufbauen \\
& - Vorausschauend denken und handeln \\
& - Interdisziplinär Erkenntnisse gewinnen und handeln \\
& - Gemeinsam mit anderen planen und handeln können \\
& - An Entscheidungsprozessen partizipieren können \\
& - Andere motivieren können, aktiv zu werden \\
& - Die eigenen Leitbilder und die anderer reflektieren können \\
Eigenständiges Handeln & - Selbständig planen und handeln können \\
& - Empathie und Solidarität für Benachteiligte zeigen können \\
\end{tabular}

verändernden Gesellschafts- und Wirtschaftssysteme heute und in Zukunft benötigen. „Dies bedeutet über die Vermittlung von Lesen, Schreiben und Rechnen hinauszugehen und sich auf Lernumgebungen und neue Zugänge für ein Lernen für größere Gerechtigkeit, soziale Ausgewogenheit und globale Solidarität zu konzentrieren. Bei Bildung muss es darum gehen, zu lernen, auf einem bedrohten Planeten zu leben." (UNESCO 2015b, 3, eigene Übersetzung). Um in Österreich die Ziele der UN-Dekade umzusetzen, wurde 2008 im Ministerrat die „Österreichische Strategie zur Bildung für nachhaltige Entwicklung" beschlossen. Mehrfach wird darin auch auf notwendige Veränderungen im Bildungssystem (Lehrpläne, Lehreraus- und -weiterbildung) hingewiesen (BMUKK et al. 2008).

Aufgrund der anerkannten Bedeutung von Bildung für nachhaltige Entwicklung (BNE) hat sich die internationale Staatengemeinschaft 2012 (Rio+20) darauf geeinigt, dieses Thema nach dem Ende der Dekade als Weltaktionsprogramm weiterzuführen. Als Ziel von BNE wird dabei die Transformation der Gesellschaft definiert. Dadurch soll ein „Übergang zu nachhaltigeren Wirtschaftssystemen und Gesellschaften" ermöglicht und erreicht werden, dass sich Menschen für „eine gerechtere, friedlichere, tolerantere, ganzheitlichere, sicherere und nachhaltigere Welt" einsetzen (UNESCO 2014, 12). In dem von den Vereinten Nationen sehr weit gefassten Verständnis von BNE sieht de Haan (2008) die Gefahr, dass alles was für das Erreichen einer positiven gesellschaftlichen Entwicklung wichtig erscheint, unter dem Begriff BNE zusammengefasst wird und damit zu einer Überfrachtung dieses Unterrichtsprinzips führt. BNE kann heute als gemeinsames Dach über den beiden bereits wesentlich weiter zurückreichenden Bildungskonzeptionen Umweltbildung und Globales Lernen betrachtet werden (Schuler \& Kanwischer 2013; Schreiber et al. 2010; Scheunpllug 2008). Dabei sehen die Vertreter/ innen des Globalen Lernens jedoch die Gefahr, dass im Zusammenhang mit der Nachhaltigkeitsdiskussion ökologische Probleme vorrangig beachtet werden, während der Aspekt der weltweiten Gerechtigkeit ${ }^{1}$ vernachlässigt wird (Scheunpflug 2008).

Als zentrales Ziel für eine erfolgreiche Umsetzung von BNE in der Praxis wird von De Haan (2008) die Vermittlung von Gestaltungskompetenz verstanden. Basierend auf dem Referenzrahmen für Schlüsselkompetenzen der OECD (2005) mit seinen drei Kompetenzkategorien definiert De Haan insgesamt zehn Teilkompetenzen (siehe Tabelle 1). Davon erscheinen für das Projekt „AustrIndia-4QOL“ vor allem die Teilkompetenzen „Weltoffen und neue Perspektiven integrierend Wissen aufbauen“, „Gemeinsam mit anderen planen und handeln können“, „Die eigenen Leitbilder und die anderer reflektieren können“ und „Empathie und Solidarität für Benachteiligte zeigen können“ von Bedeutung (De Haan 2008, 32). Der GWK-Unterricht hat für die Förderung interkultureller Kompetenz die Fähigkeit zum Perspektivenwechsel und zum Hinterfragen des eigenen Weltbildes zu vermitteln (Schrüfer 2010).

Einen fehlenden Konsens über die zu vermittelnden Kompetenzen im Zusammenhang mit dem Konzept des Globalen Lernens kritisieren Lang-Wojtasik und Scheunpflug (2005). Scheunpflug und Schröck (2002) definieren drei Dimensionen Globalen Lernens (Sach-, Zeit- und Sozialdimension) für die Vorbereitung Jugendlicher auf das Leben in einer Welt, die von Widersprüchen gekennzeichnet ist. Darauf aufbauend formuliert Asbrand (2008) Forderungen zur Konzeption von Angeboten für Globales Lernen. Erstens müssen für das Erlernen eines adäquaten Umgangs mit Wissen

\footnotetext{
Eine Diskussion unterschiedlicher Definitionen von Gerechtigkeit würde den Rahmen dieses Artikels sprengen. Für eine umfangreichere Auseinandersetzung u.a. mit dem für den vorliegenden Artikel bedeutsamen Aspekt der globalen Gerechtigkeit sei z. B. auf Hahn (2009), Sedmak (2014) oder Höffe (2015) verwiesen.
} 
und Nichtwissen unterschiedliche Perspektiven berücksichtigt und kontroverse Wertorientierungen und Problemlösungen diskutiert werden. Es genügt nicht, vermeintlich sicheres Wissen zu präsentieren. Zweitens sind Lernarrangements so zu gestalten, dass Jugendliche auf den Umgang mit Unsicherheiten und Ungewissheit vorbereitet werden. Handlungskompetenz ist abhängig von den erlernten Strategien zum Umgang mit Unsicherheiten. Drittens sind Kompetenzen für den Umgang mit sozialen und kulturellen Disparitäten zu erwerben, wobei dazu vor allem internationale Partnerschaften geeignet sind, die auf einer gleichberechtigten Zusammenarbeit mit einer gemeinsamen Handlungspraxis aufbauen (Asbrand 2008).

\subsection{Empirische Erkenntnisse aus verschiedenen Unterrichts- und Forschungsprojekten zur interkulturellen Zusammenarbeit}

\subsubsection{Interkulturelles Lernen - Veränderungen von Wissen, Einstellungen und Handlungen}

Die verstärkte Förderung der Zusammenarbeit zwischen österreichischen und internationalen Schulen ist das Ziel des Interkulturellen Zentrums in Wien, einem Verein der sich schwerpunktmäßig der Unterstützung interkultureller Bildungsprojekte widmet. Bezugnehmend auf entsprechende Literatur und die Erfahrungen bestehender Schulpartnerschaften, werden in einem Handbuch zehn Empfehlungen für nachhaltige Schulpartnerschaften genannt. Davon erscheinen für das Forschungsvorhaben „AustrIndia-4QOL“ vor allem die Empfehlungen zur partizipativen Einbindung der Schüler/innen, zur Zusammenarbeit auf Augenhöhe und zur thematischen Zusammenarbeit zentral: „Im Mittelpunkt steht das gemeinsame Lernen, nicht das Lernen über ,den anderen “" (Interkulturelles Zentrum 2014, 32).

Budke (2013) weist in diesem Zusammenhang darauf hin, dass die im Umgang mit fremden Kulturen oft auftretenden Simplifizierungen, Klischees und Stereotype im Rahmen des GWK Unterrichtes nur sehr schwer durch ein realitätsnäheres Verständnis der Wirklichkeit ersetzt werden können. Sie fordert deshalb, den Fokus verstärkt auf das Hinterfragen der Konstruktionsprinzipien zu legen, denen verschiedene Kulturdefinitionen zu Grunde liegen und den Schülerinnen und Schülern die Problematik solcher Konstrukte aufzuzeigen. Nur dann kann ein Perspektivenwechsel gelingen (vgl. auch Schrüfer \& Obermaier 2014). Bereits die Untersuchungen von Schmidt-Wulffen (2001) zu den Alltagsvorstellungen von deutschen und ghanaischen Kinder und Jugendlicher über das Leben in Ghana bzw. in Deutschland haben gezeigt, dass durch die gängige Unterrichtspra- xis, bei der die Auseinandersetzung mit fremden Ländern meist über Informationen aus dem Schulbuch, über digitale Medien oder durch die Lehrperson erfolgt, Klischees und unzulässige Stereotype nicht ausgeräumt werden. Bestehende oder vermeintliche Unterschiede werden mit einem derartigen Unterricht oft im Gegenteil noch verstärkt (Schrüfer 2010). Stereotype bestehen auch dann weiter, wenn deren Grundlagen widerlegt sind (Reinfried 2015). Gerade im Vergleich mit Entwicklungs- oder Schwellenländern bestehe die Gefahr, dass die eigene Kultur „als intellektuell und materiell überlegen gesehen" wird (Schrüfer 2004, 46).

Zur Frage, ob die oft mit einem hohen finanziellen und organisatorischen Aufwand verbundenen klassischen Schüleraustauschprogramme ein differenzierteres Verständnis eines fremden Landes bewirken, geben Thomas und Perl eine Übersicht für Deutschland. Sie bezeichnen das Vorhandensein wissenschaftlicher Evaluationen zu diesem Thema als "national wie international eher marginal" (Thomas \& Perl 2010, 288). Die von ihnen angeführten Studien (u. a. Thomas et al. 2007) zeigen jedoch selbst längerfristig betrachtet einen positiven Einfluss, u. a. auf die Persönlichkeitsentwicklung der Teilnehmer/innen sowie deren interkulturelles Verständnis. Ein differenzierteres Bild zeigt eine Studie von Jones (2014) über die Einstellungen von US-amerikanischen Austauschstudent/innen. Während durch den Austausch zwar Ängste gegenüber „den Anderen“ abgebaut werden, kam es nicht zu einem verstärkten Gefühl einer gemeinsamen internationalen Gesellschaft. Vielmehr wurde eine verstärkte Betonung der eigenen Identität festgestellt. Jones (2014, 693) spricht deshalb von einer enlightened form of nationalism. Auch Lang-Wojatsik und Scheunplug (2002) halten fest, „dass eine Reise als solche in keiner Weise interkulturelles Lernen oder den Abbau von Fremdenfeindlichkeit garantiert. Bedeutsam sind die pädagogischen Rahmenbedingungen und deren Durchführung". Speziell Reisen mit entwicklungspolitischem Hintergrund (über)fordern sehr oft die Reisenden, sodass interkulturelles Lernen auf der Strecke bleibt (Lang-Wojatsik \& Scheunpflug 2002, 28). In den von Asbrand (2009) durchgeführten qualitativen empirischen Erhebungen zeigten Schüler/innen, die an einer Begegnungsreise nach Afrika teilgenommen hatten, deutlich stärkere Stereotype gegenüber Menschen in Entwicklungsländern als Schüler/innen, die an ihrer eigenen Schule einen Weltladen betreiben und sich eher als gleichberechtigte wirtschaftliche Partner der Produzent/innen sehen. Entscheidender als ein tatsächlicher physischer Kontakt ist demnach, dass gemeinsame Schulprojekte auf Augenhöhe stattfinden. Eine z.B. vorrangig auf das Sammeln von Spenden ausgerichtete Partnerschaft mit einer Schule 
aus einem Entwicklungsland führt fast zwangsläufig zu einen asymmetrischen Weltbild, in dem die Partner als defizitär und hilfsbedürftig kategorisiert werden, weshalb in Partnerschaftsprojekten „auf das Spendensammeln für die Partnerorganisationen im Süden, d.h. auf das Helfen, konsequent zu verzichten" sei (Asbrand 2009, 235 f.). In der von Asbrand durchgeführten Studie zeigt sich zudem, dass ein hohes Maß an Wissen und eine positive Einstellung nicht zwangsläufig zu einem entsprechenden Handeln führt. Die in der Schule vermittelten ethisch-moralischen Werte bleiben für die Schüler/innen theoretisch, da sie keine realen Anknüpfungspunkte für ihren Alltag finden können. Sie suchen vielmehr nach Begründungen bzw. Entschuldigungen, wieso sie selbst keine konkreten Handlungen setzen.

Auch ein Austausch über digitale Medien im Rahmen des GWK-Unterrichts an Schulen führt nicht unmittelbar zu einem Abbau von Kategorisierungen und hierarchischen Beziehungen zwischen Jugendlichen aus unterschiedlichen Ländern. Dieses Ergebnis brachte eine von Höhnle (2014) durchgeführte Studie, im Zuge derer sich Schulklassen aus Deutschland, den USA und Indien zum Thema Klimawandel ausgetauscht haben. Bei den Abschlusserhebungen zeigten vor allem die Schüler/innen aus Deutschland eine gewisse Überheblichkeit gegenüber den Ansichten der indischen und US-amerikanischen Schüler/innen. Im Gegensatz dazu bewerteten die indischen Schüler/innen ihr Land als hierarchisch den Industriestaaten untergeordnet. Höhnle fordert deshalb für eine erfolgreiche Umsetzung von internationalen Online Projekten ausreichende Reflexionsphasen unter Leitung der Lehrkraft, um längerfristig einen Perspektivenwechsel zu ermöglichen (Höhnle 2014).

\subsubsection{Austausch über digitale Medien}

Dank der technologischen Entwicklung kann heute über digitale Medien eine Zusammenarbeit mit Schülerinnen und Schülern aus einem anderen Land mit relativ geringem organisatorischem und vor allem finanziellem Aufwand umgesetzt werden. Bei Projekten zur internetbasierten Kommunikation in multinationalen Gruppen zur Steigerung der Fremdsprachenkompetenzen wird oftmals als Nebenprodukt von einer Stärkung der interkulturellen Kompetenz durch den Austausch mit Personen aus einem anderen Land bzw. einem anderen kulturellen Hintergrund ausgegangen. Helm kritisiert, dass in diesem Zusammenhang aber meist nur oberflächliche Gespräche über Alltagsthemen stattfinden, die kaum Konfliktpotential beinhalten. Sie lässt deshalb in ihrer eigenen Studie Student/innen aus unterschiedlichen Nationen kontroverse Themen über Videokonferenzen diskutieren.
Bei der Evaluation führen die Teilnehmer/innen als wichtigsten Punkt an, gelernt zu haben, keine Generalisierungen vorzunehmen oder über Personen zu urteilen, ohne sie zu kennen und mit ihnen gesprochen zu haben (Helm 2013). Lamy und Goodfellow (2010) weisen jedoch auf mögliche Schwierigkeiten hin, die bei Projekten mit einem Austausch über digitale Medien zu berücksichtigen sind. Die teilweise sehr verschiedenartigen Kommunikationsweisen der Teilnehmer/innen, unterschiedliche Werthaltungen oder vorherrschende Ideologien können eine Diskussion über sensible Themen unter Umständen sogar verunmöglichen.

Dennoch erscheinen die heutigen technischen Möglichkeiten der Kommunikation mit Menschen aus unterschiedlichsten Regionen der Welt auch für den Unterricht in GWK als große Chance, indem Informationen über die Lebensweise und Einstellungen dieser Menschen nicht nur aus Schulbüchern o. ä., sondern durch einen direkten Kontakt aus erster Hand bezogen werden können. Das Lernen über Menschen in anderen Regionen kann erweitert werden zu einem gemeinsamen Lernen mit einem bidirektionalen Informationsfluss. Neben kognitiven Lernzielen wird durch den Austausch zwischen Jugendlichen über Ländergrenzen hinweg auch die affektive Lernkomponente gefördert (Schleicher 2006). Trotz der zumindest in den Industriestaaten intensiven Nutzung des Internets durch Jugendliche findet kaum eine direkte Kommunikation mit Gleichaltrigen aus fernen Ländern statt. Mit einem gezielten Einsatz sozialer Medien im Unterricht könnte die Möglichkeit zur Kooperation über Landesgrenzen hinweg gefördert werden (Kammerl 2009) und den sozialen Netzwerken eine Funktion abseits des persönlichen „Identitätsmanagements" zukommen (Schreiber et al. 2010, 27). Auf diese und weitere Vorteile von Facebook für internationale Kooperationen im tertiären Bildungsbereich aber auch auf die Grenzen dieses Mediums verweisen z. B. McCarthy (2012) oder Wang et al. (2012). Höhnle erhob, wie Schüler/innen Facebook als mögliche Kommunikationsplattform für Schulprojekte beurteilen. Positiv bewertet wird von den Jugendlichen dabei der Umstand, dass sie sich im Vergleich zu speziellen Lernplattformen öfters online auf Facebook aufhalten und dadurch intensivere Diskussionen erwarten. Negativ erwähnen sie eine dadurch entstehende Vermischung von Freizeit und Schule und damit die Befürchtung, dass die Diskussionen auf Facebook zu wenig ernsthaft betrieben würden (Höhnle 2014).

In der Realität besteht immer noch ein großes Gefälle zwischen den Industrie- und Entwicklungsländern in Bezug auf deren Zugang zum Internet. Auch lassen sich besonders in Regionen mit einer allgemein geringeren Internetnutzung große Unterschiede zwi- 
schen verschiedenen gesellschaftlichen Schichten beobachten. Damit besteht die Gefahr, dass durch den unterschiedlichen Zugang zu diesen neuen Medien bestehende Ungleichheiten weiter verstärkt werden (Kammerl 2009). Die Erwartung, bei einer Kooperation über digitale Medien mit Schulen aus Entwicklungsländern einen repräsentativen Querschnitt der Bevölkerung in diesen Ländern zu erreichen, kann deshalb sicher nicht erfüllt werden.

\subsubsection{Der transdisziplinäre Aspekt}

Im Forschungsprojekt „AustrIndia-4QOL“ werden die Schüler/innen aus Österreich und Indien den Lernprozess in einem hohen Maße selbst mitgestalten, indem sie nicht an vorgegebenen Themenbereichen oder Forschungsfragen arbeiten, sondern selbstständig Themenbereiche wählen und dazu passende Forschungsfragen und Hypothesen entwickeln. Eine vergleichbare Vorgangsweise wurde auch in den am Institut für Geographie der Universität Innsbruck in den letzten Jahren durchgeführten fachdidaktischen Forschungsprojekten gewählt. Dabei beschäftigten sich z. B. Jugendliche aus verschiedenen Regionen des Alpenraumes mit ihren „Lebensqualitätsvorstellungen“ vor dem Hintergrund der Nachhaltigkeit. Die Jugendlichen bzw. deren Vorstellungen von Lebensqualität wurden nicht nur aus der Sicht von Wissenschaftlerinnen und Wissenschaftlern erforscht. Vielmehr agierten die Schüler/innen selbst aktiv als Forscher/innen, um die Sichtweisen Jugendlicher zu ergründen und gemeinsam Visionen für eine nachhaltige Lebensqualität in der Region zu entwickeln. Ziel war es somit, in einem weiteren Schritt bei den Jugendlichen Handlungsbereitschaft für eine nachhaltige Gestaltung der Zukunft zu fördern. Bei den im Rahmen dieser Forschungen vorgenommenen Erhebungen zeigt sich, dass bei vielen Jugendlichen durch die Teilnahme am Projekt ökologische Faktoren für die persönliche und allgemeine Lebensqualität in der Region an Bedeutung gewinnen (Keller \& Oberrauch 2013; Keller \& Schober 2011; Oberrauch et al. 2014; Oberrauch \& Keller in Vorbereitung). Diese Studien liefern auch einen Einblick in das Verständnis von Jugendlichen zum Thema intragenerationale Gerechtigkeit. Bei der von Oberrauch et al. (2014) durchgeführten Untersuchung definieren $50 \%$ der befragten Jugendlichen ihre eigene Lebensqualität über einen Vergleich mit Lebensqualität in anderen Regionen. Zwar greifen sie dabei meist auf übliche Stereotype (z. B. Afrika als Kontinent der Armut) zurück, zeigen damit aber, dass ihnen ihr eigener weltweit privilegierter Wohlstand bewusst ist. Von $10 \%$ der Schüler/innen wird zudem explizit im Sinne von intragenerationaler Gerechtigkeit hohe Lebensqualität für alle Menschen dieser Erde gefordert.
Ebenfalls direkt in die Forschung eingebunden wurden Jugendliche in der von Leitner et al. (2011) durchgeführten Studie „my life - my style - my future“. Sie haben dabei mit Hilfe einer selbst erstellten Umfrage an ihrer Schule die Lebensstile von Schülerinnen und Schülern im Alter zwischen 10 und 19 Jahren erforscht. Indem jeweils eine Schulklasse aus Japan sowie aus Kamerun ebenfalls diese Fragebögen beantwortete, wurde auch in diesem Projekt eine länderübergreifende Zusammenarbeit zumindest in Ansätzen verwirklicht. Diese Komponente wird im Forschungsprojekt „AustrIndia4QOL“ weiter ausgebaut, indem nicht nur das Erforschen der jeweils anderen Lebensumstände und Sichtweisen das Ziel darstellt, sondern das gemeinsame Forschen an einem inhaltlichen Thema.

Die Erkenntnisse und Empfehlungen aus den genannten Studien fließen in die Konzeption des Forschungsprojekts „AustrIndia-4QOL“ ein. Die wissenschaftliche Begleitung des Projekts verfolgt das Ziel, die empirische Datengrundlage im Rahmen von BNE zu verbreitern und zu einem besseren Verständnis offener Fragen beizutragen.

\section{Das Forschungsprojekt „AustrIndia- 4QOL“}

\subsection{Ausgangspunkt}

Im Projekt „AustrIndia-4QOL“ arbeiten Schüler/innen der Day Star School in Manali im Bundesstaat Himachal Pradesh in Nordindien und des BRG und BORG Feldkirch in Vorarlberg gemeinsam an geographischen Fragestellungen. Durch die Zusammenarbeit von Jugendlichen aus zwei Ländern mit großen sozio-ökonomischen Unterschieden, können Themen nachhaltiger Entwicklung aus multiperspektivischer Sicht behandelt werden. Die eigene Fähigkeit zum Perspektivenwechsel und zur Zusammenarbeit über Länder- und Kulturgrenzen hinweg ist eine wichtige Kompetenz für die Bewältigung globaler Herausforderungen in der Zukunft (De Haan 2008). Interkulturelles Lernen ist in den Lehrplänen sämtlicher allgemein bildenden Schulen als Unterrichtsprinzip verankert, um das gegenseitige Verständnis zu stärken, Unterschiede und Gemeinsamkeiten zu erkennen sowie den Abbau von Vorurteilen zu fördern (BMBF 2015). Es wäre in Bezug auf das Projekt „AustrIndia-4QOL“ jedoch eine unzulässige Vereinfachung, von einem einheitlichen indischen bzw. österreichischen Kulturraum auszugehen. Neben ethnisch-kulturellen Unterschieden zwischen den Schülerinnen und Schülern aus Österreich und Indien sind diese Jugendlichen auch innerhalb ihrer Region in unterschiedliche kulturelle Milieus (Einkommensschich- 

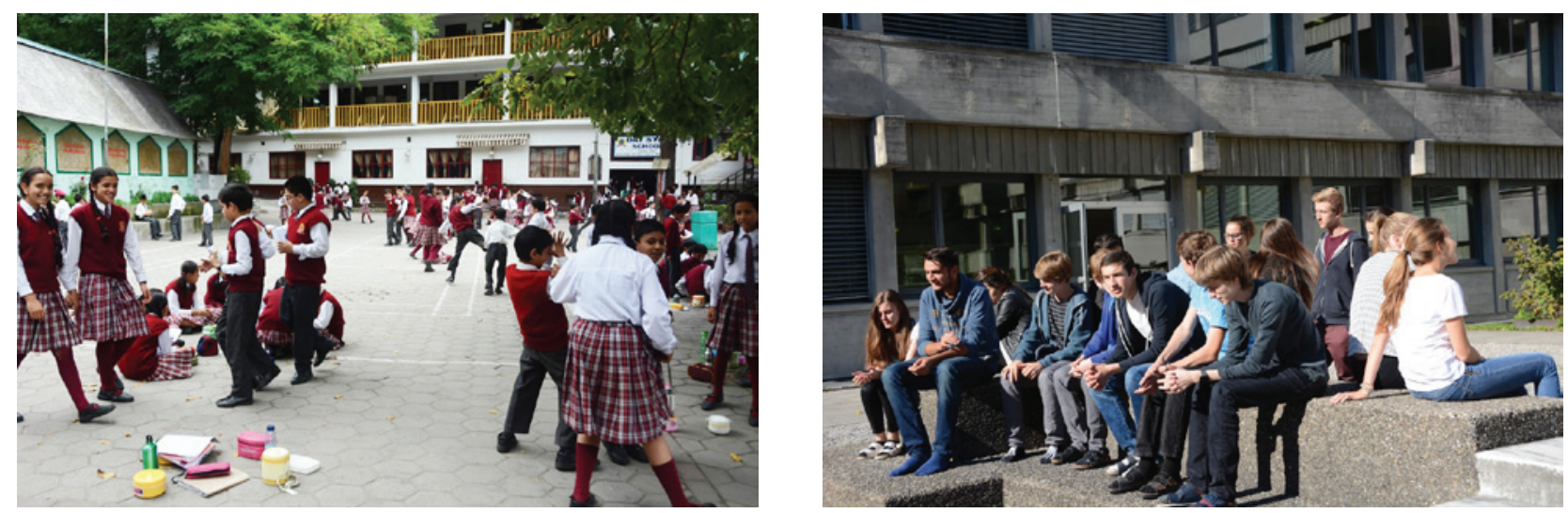

Abb. 1: Die am Projekt teilnehmenden Schulen aus Indien und Österreich. Eigene Aufnahmen

ten, Religionsgruppen, etc.) eingebunden, die ihre Alltagsrealität beeinflussen. Unter dem Begriff Kultur sind nicht nur Beschreibungen einer bestimmten Lebensweise, sondern auch Selbstrepräsentationen von Gruppen und Fremdrepräsentationen von außen auf diese Gruppe zu verstehen. Diese Vielschichtigkeit des Begriffs Kultur beschreibt Nohl (2010) mit seinem Konzept der „kollektiven Zugehörigkeit“. Wenn im Folgenden vereinfachend von einer „länderübergreifenden Zusammenarbeit" die Rede ist, ist zu berücksichtigen, dass dabei Jugendliche zusammenarbeiten, deren Lebensrealitäten große Unterschiede aufweisen. Die Schüler/innen aus Österreich arbeiten im Projekt „AustrIndia-4QOL“ mit Jugendlichen aus einem Land zusammen, das auf der einen Seite in der Lage ist, erfolgreiche Raumfahrtmissionen zum Mars durchzuführen, in dem aber auf der anderen Seite rund 600 Mio. Einwohner/innen keinen Zugang zu Toiletten haben (WHO \& UNICEF 2014). Für die indischen Schüler/innen bedeutet dieses Forschungsprojekt eine Kooperation mit Gleichaltrigen aus einem Land, das weltweit nicht nur einen der höchsten materiellen Lebensstandards aufweist, sondern das auch bei Erhebungen zur Lebensqualität überdurchschnittlich gut abschneidet (z. B. OECD o. J.). Wie werden die Schüler/innen in den gemeinsamen Diskussionen mit dem großen materiellen Wohlstandsgefälle zwischen den beiden Ländern umgehen und wie mit der Tatsache, dass ein weltweiter Anstieg des pro Kopf Verbrauchs an Ressourcen auf das Niveau in Österreich drastische Folgen für die Umwelt nach sich ziehen würde?

\subsection{Zielsetzungen des Forschungsprojekts „AustrIndia-4QOL“}

Bildung für Nachhaltige Entwicklung wird von der UNESCO als Voraussetzung definiert, um „informierte Entscheidungen zu treffen und verantwortungsbewusst zum Schutz der Umwelt, für eine be- standsfähige Wirtschaft und eine gerechte Gesellschaft für aktuelle und zukünftige Generationen zu handeln und dabei die kulturelle Vielfalt zu respektieren" (UNESCO 2014). Das Projekt „AustrIndia-4QOL“ sieht sich diesen von der UNESCO formulierten Zielen verpflichtet und beabsichtigt eine Stärkung der Handlungskompetenz von Jugendlichen im Sinne dieser Ziele. Die teilnehmenden Jugendlichen forschen gemeinsam zum Thema "Nachhaltigkeit unter dem Aspekt der intragenerationalen Gerechtigkeit". Das Forschungsprojekt versucht dabei primär Antworten auf folgende Forschungsfragen zu finden:

1. Welche Auswirkungen hat der unterschiedliche sozio-ökonomische Hintergrund auf die Vorstellungen von Lebensqualität bei Jugendlichen aus Vorarlberg und der Kullu-Region in Nordindien? Unter Vorstellungen von Lebensqualität werden folgende Aspekte subsumiert: persönliche Lebensqualität, Lebensqualität im globalen Maßstab und Außensicht auf die Lebensqualität der „Anderen“.

2. Inwiefern verändern sich durch die partizipative Zusammenarbeit gegenseitige Stereotype?

3. Wie wirken sich die gewählten Lernsettings auf das Bewusstsein und die Handlungsbereitschaft Jugendlicher für eine nachhaltige und global gerechte Lebensweise aus?

4. Welche Konsequenzen ergeben sich durch diese Zusammenarbeit auf die Kompetenz von Jugendlichen, multiperspektivische Sichtweisen bei der Suche nach Lösungsansätzen für globale Herausforderungen mit einzubeziehen?

\subsection{Geplanter Projektablauf}

Das Forschungsprojekt „AustrIndia-4QOL“ beginnt mit der Durchführung eines Pretests, bestehend aus einem Freewriting zum Thema „Lebensqualität" und einem Online-Fragebogen. Damit können die bei den Jugendlichen vorhandenen Präkonzepte erfasst wer- 
den, die entsprechend der didaktischen Theorie des Conceptual Change bei der Aneignung von Wissen einen entscheidenden Einfluss haben (z. B. Reinfried 2007; Schuler 2013). Dass durch die Anwendung dieser didaktischen Theorie ein signifikant höherer Wissenszuwachs erreicht werden kann, zeigen z. B. Reinfried et al. (2010). Aber auch im Zusammenhang mit interkulturellem Lernen erscheint die Berücksichtigung der Conceptual Change Theorie zentral. Die Abgrenzung von Räumen und Kulturen erfolgt im Normalfall nicht nur anhand objektiver Kriterien sondern genauso durch die von den Betrachtern selbst konstruierten Bewertungsmuster. Diese vorhandenen Muster bewusst zu machen, ist entscheidend, um Vorurteile abzubauen und ein besseres interkulturelles Verständnis zu fördern (Hofmann \& Ulrich-Riedhammer 2014; Nohl 2010).

Wie in Abbildung 2 ersichtlich, setzen sich die Jugendlichen in der ersten Phase des Projekts im Oktober 2015 mit ihren eigenen Vorstellungen von Lebensqualität auseinander und erforschen als Vergleich die Vorstellungen von anderen Jugendlichen aus Österreich und Indien. Die dabei zu Tage tretenden Unterschiede bzw. Gemeinsamkeiten werden anschließend in Kleingruppen mit Gleichaltrigen aus dem jeweils anderen Land diskutiert. In der zweiten Projektphase bekommen die Jugendlichen die Möglichkeit, in Kleingruppen selber Themen aus dem Bereich intragenerationale Gerechtigkeit zu definieren, Forschungsfragen zu entwickeln, eine geeignete Methodik zu wählen und durch eigene Forschungstätigkeit Antworten auf die gestellten Fragen zu finden. Diese Aneignung von Wissen in aktiven und selbstgesteuerten Prozessen entspricht einer moderat konstruktivistischen Vorgangsweise des Lernens, bei dem die Lehrenden ihr Wissen dafür nützten, Impulse, Steuerung und Beratung anzubieten. Lernende werden nicht mehr als Rezipient/innen von Wissen, sondern als eigenständig tätige Konstrukteur/innen verstanden (Rhode-Jüchtern 2011; Schmidt-Wulffen 2008). Ein anschließender Diskussionsprozesses der Ergebnisse mit einer Kleingruppe aus dem jeweils anderen Land soll in einer gemeinsamen Abschlusserklärung münden. Dadurch festigen die Jugendlichen ihre neu gewonnenen Konzepte. Je nach selbst gewählter Fragestellung werden die Schüler/innen feststellen, dass eine allgemeingültige und abschließende Antwort aufgrund der hohen Komplexität gar nicht gefunden werden kann, ein Handeln jedoch trotz dieser Unsicherheit notwendig erscheint. Wie Rhode- Jüchtern (2009) am Beispiel der Klimawandeldiskussion aufzeigt, führt bereits das Wissen um die Konstruktionsprinzipien hinter verschiedenen Argumentationsmustern zu einem wichtigen Erkenntnisgewinn der Schüler/innen. Entsprechend den Erkenntnissen von
Höhnle (2014) steht am Ende jeder Projektphase eine Reflexionsphase, um die Erfahrungen und Erkenntnisse gemeinsam zu besprechen.

Für die Kommunikation der indischen und österreichischen Schüler/innen werden Videokonferenzen über Skype sowie die social media Plattform Facebook zum Einsatz kommen. Damit wird sowohl eine asynchrone (Facebook) als auch synchrone (Skype) Kommunikation zwischen den Schülerinnen und Schülern ermöglicht (vgl. Höhnle 2014). Fünf der 29 Schüler/innen der indischen Schulklasse verfügen über keinen Facebook Account da sie kaum Zugriff auf das Internet haben. Für jene drei Schüler/innen der österreichischen Schulklasse, die ihren Account ausschließlich aufgrund dieses Schulprojektes eingerichtet haben, sind im Gegensatz dazu andere Beweggründe (Datenschutz etc.) ausschlaggebend. Dank der weiten Verbreitung von Smartphones in Indien gehört für die restlichen am Projekt „AustrIndia-4QOL“ teilnehmenden Jugendlichen die Kommunikation über soziale Medien zum Alltag. Der hohe Bekanntheitsund Verwendungsgrad von Facebook bei Jugendlichen bildete auch die Entscheidungsgrundlage, diese Kommunikationsplattform klassischen Lernplattformen wie z. B. Moodle vorzuziehen.

Im Oktober 2016 ist zudem ein direkter Besuch von ca. 15 indischen Schülerinnen und Schülern in Österreich vorgesehen. Im Rahmen dieses Besuchs sind gemeinsame Workshops geplant, in denen sich die Schüler/innen nochmals vertiefend mit den jeweiligen Themen auseinandersetzen und im Rahmen einer öffentlichen Abschlusspräsentation ihre Ergebnisse vorstellen. Basierend auf der Evaluation dieser ersten Forschungsphase und um eventuell auftretende Schwächen bereinigt, wird der Projektablauf im Schuljahr 2016/2017 ein zweites Mal mit einer neuen Schüler/innengruppe durchgeführt, womit auch die Datengrundlage verbreitert werden kann.

\section{4 Überlegungen zu Stichprobenauswahl und Forschungsmethodik}

Die Jugendlichen der am Projekt teilnehmenden Schulklassen stammen aus bzw. aus der unmittelbaren Umgebung der beiden größenmäßig vergleichbaren Kleinstädte Feldkirch (ca. 30000 Einwohner) und Manali (ca. 43000 (sub-district Manali) Einwohner) (Government of India 2011). Die Schule in Manali bietet für das Projekt „AustrIndia-4QOL“ u.a. den Vorteil, dass entsprechend den Leitlinien dieser englischsprachigen Privatschule gezielt Schüler/innen aus allen ethnischen, religiösen und sozialen Gruppen aufgenommen werden. Damit wird eine für diese Region annähernd repräsentative Durchmischung der Jugendlichen erreicht und es kommt keine „Voraus- 


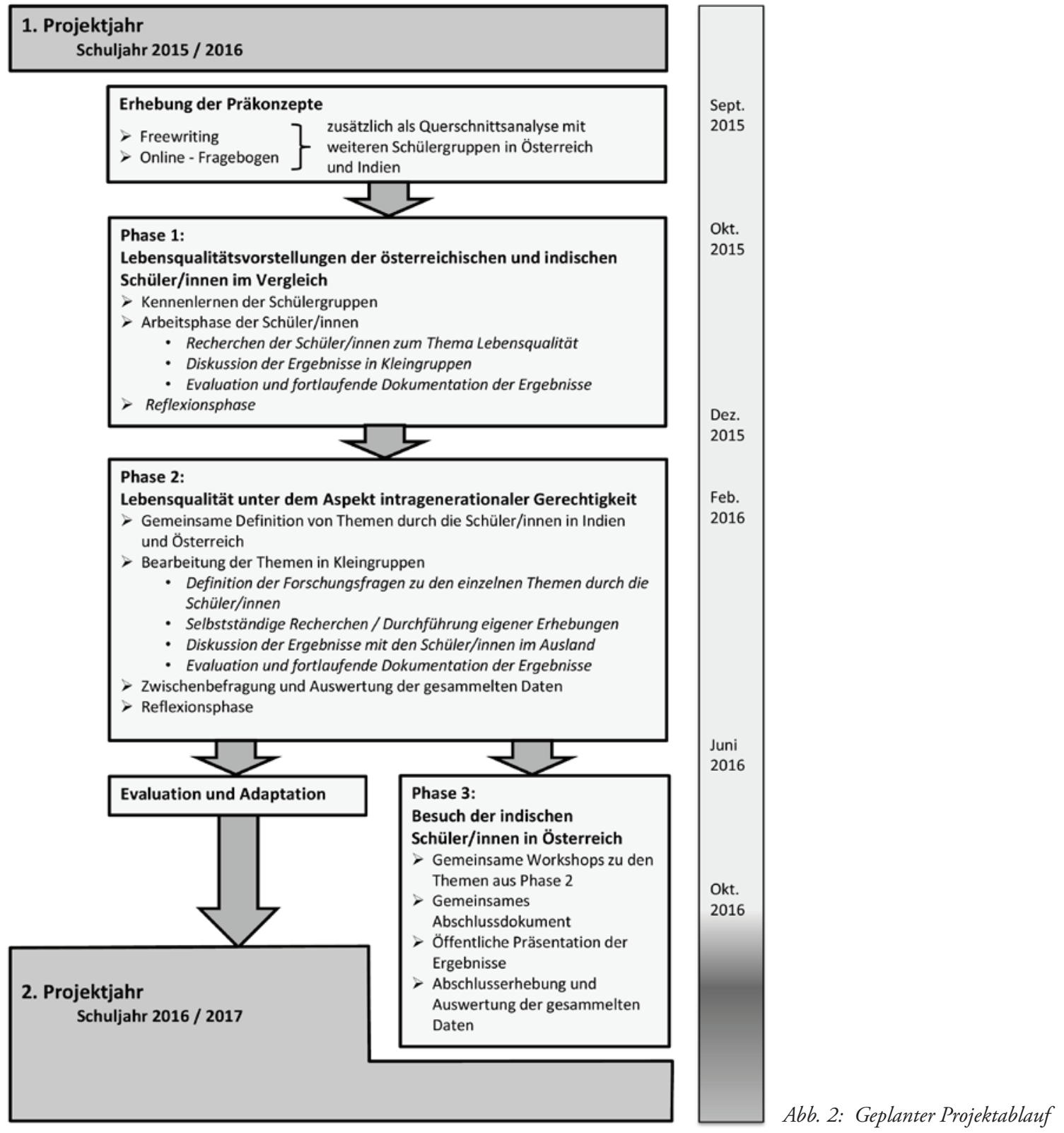

wahl“ bestimmter Bevölkerungsschichten zum Tragen. Menschen aus Indien sind in Vorarlberg nicht als klassische Migrant/innengruppe vertreten und diesbezügliche persönliche Kontakte der Vorarlberger Schüler/innen eher unwahrscheinlich. Da Österreich auf internationaler Ebene wenig präsent ist, kann davon ausgegangen werden, dass die indischen Schüler/innen generell noch kaum mit medialen Bildern aus und über Österreich konfrontiert worden sind. Dadurch sind relativ wenige Vorurteile über die jeweils andere Gruppe zu erwarten. Als Altersgruppe wurden 16-17 jährige Schüler/innen gewählt, da sich Jugendliche in diesem Alter vielfach sehr intensiv mit Fragen zu ihrer eigenen Zukunft beschäftigen, weshalb von einem entsprechenden Interesse ihrerseits ausgegangen werden kann. Die bereits vorhandene Reife ist auch eine Voraussetzung, um als Abschluss des Projekts den persönlichen Austausch einer Schülergruppe durch- führen zu können. Die in dieser Altersstufe bereits vorhandenen Englischkenntnisse der österreichischen Schüler/innen sind notwendig, um eine inhaltliche Zusammenarbeit über social media mit Gleichaltrigen aus Indien in der Praxis durchführen zu können. Diesbezüglich sind die indischen Schüler/innen im Vorteil, da an der betreffenden Schule der Unterricht generell auf Englisch gehalten wird.

Für die Beantwortung der ersten Forschungsfrage (siehe oben) wird der Pretest mit weiteren Schülergruppen in Vorarlberg sowie in der indischen Kullu-Region durchgeführt, um für diese Querschnittsstudie eine entsprechende Stichprobengröße (ca. 120 Jugendliche pro Land) zu erreichen, und Aussagen über die Lebensqualitätsvorstellungen von Jugendlichen in diesen Regionen treffen zu können. Bei diesen Befragungen der Jugendlichen über einen Online-Fragebogen, sowie durch die Durchführung von Freewritings werden 
entsprechend eines Mixed Methods Ansatzes jeweils parallel quantitative und qualitative Daten erhoben. Für die Auswertung werden die qualitativen Daten im Zuge einer qualitativen Inhaltsanalyse mit Hilfe der Software MaxQDA codiert und mit den quantitativen Daten für eine statistische Analyse zusammengeführt (Kuckartz 2014b). Damit kann untersucht werden, ob tatsächlich signifikante Unterschiede in den Einstellungen von indischen und österreichischen Jugendlichen existieren oder ob die Differenzen innerhalb der beiden Schülergruppen bedeutender sind.

Um Antworten auf die Forschungsfragen zwei bis vier geben zu können, d.h. mögliche Veränderungen von Einstellungen durch das gewählte Lernsetting zu erfassen, wird zudem mit insgesamt ca. 50 Schüler/innen aus Österreich und ca. 70 Schüler/ innen aus Indien (auf zwei Schuljahre verteilt) eine Längsschnittsstudie durchgeführt. Dazu wird die Datenerhebung während des Forschungsprojekts im Sinne eines PrePosttest Vergleichs wiederholt. Diese Pre- und Posttests werden zudem durch Verfahren der laufenden Datenerhebung ergänzt. Dazu zählen die durch die Schüler/innen fortlaufend geführten Forschungstagebücher, die Aufzeichnungen der geführten Diskussionen sowie die durch die Schüler/innen erstellten Forschungsfragen und die Ergebnisse ihrer Forschungstätigkeit. Besonders um komplexe Zusammenhänge, wie die Einstellungen von Schülerinnen und Schülern besser zu verstehen, erscheint eine Kombination aus unterschiedlichen Forschungsmethoden sinnvoll (Kuckartz 2014a; Oberrauch \& Keller 2015). Da kaum vergleichbare empirische Untersuchungen vorliegen und damit die Breite der möglichen Antworten der Jugendlichen schwer fassbar ist, erscheint es sinnvoll, die qualitativen Daten in den Vordergrund zu stellen (Porst 2014). Neben der qualitativen Inhaltsanalyse der Freewritings, der offenen Fragestellungen aus den Fragebögen sowie der Forschungstagebücher mit Hilfe der Software MaxQDA (Kuckartz 2014b) dienen die quantitativen Daten dazu, für ausgewählte Aspekte auf Basis der einzelnen Jugendlichen umfangreichere Erkenntnisse durch eine Kombination aller Daten zu ermöglichen. Damit kann nicht nur die Validität der Ergebnisse erhöht, sondern auch ein tieferes Verständnis gewonnen werden (Kuckartz 2014a). Das aufwändige Setting der Studie lässt ein größeres Sample nicht zu und führt damit zu einer reduzierten Interpretierbarkeit und Generalisierbarkeit der Ergebnisse. Durch die Triangulation von den über unterschiedliche Methoden gewonnenen quantitativen und qualitativen Daten, kann dieses Problem aber zumindest teilweise entschärft und die Validität der Ergebnisse gesteigert werden.

\subsection{Erwartungen an das Projekt}

Die im Projekt „AustrIndia-4QOL“ notwendige partizipative Zusammenarbeit über Ländergrenzen hinweg und die dabei möglicherweise zu Tage tretenden unterschiedlichen Standpunkte und Auffassungen soll zudem helfen, ein Lernen aus multiperspektivischer Sichtweise zu fördern und zu einem verstärkten Infrage stellen der eigenen Sichtweise beitragen (vgl. Schuler \& Kanwischer 2011). „In einer Welt, die durch kulturelle Vielfalt, gesellschaftliche Pluralität und komplexe Globalisierungsprozesse geprägt ist, wird Perspektivenwechsel zu einer Schlüsselkompetenz" (Schreiber 2010, 39).

Neben den zahlreichen Chancen, die dieses Projekt bietet, dürfen aber auch die Herausforderungen nicht unterschätzt werden. So spannend und bereichernd eine länderübergreifende Zusammenarbeit für den Unterricht in GWK ist, wird dies auch die größten Herausforderungen mit sich bringen. Neben organisatorischen Anforderungen wie der technischen Bereitstellung einer funktionsfähigen Kommunikationsbasis über soziale Netzwerke und Videokonferenzen, der Zeitverschiebung zwischen den Ländern oder des zeitlich unterschiedlich strukturierten Schuljahres, treffen in diesem Projekt zwei völlig unterschiedliche Schulsysteme aufeinander, die sich in Bezug auf die vorherrschende Unterrichtsweise deutlich unterscheiden. Wird es möglich sein, auch an der indischen Schule eine moderat konstruktivistische Unterrichtsweise zuzulassen und wie gehen die indischen Schüler/ innen, die einen sehr lehrerzentrierten Unterrichtsstil gewohnt sind, mit der ihnen gewährten Freiheit aber auch mit der damit verbundenen Verantwortung um? Können die indischen Jugendlichen „aus der Reserve gelockt" werden und bringen sie sich aktiv in Diskussionen ein und vertreten ihre eigene Meinung? Und welche Erfahrungen werden die Schüler/innen beider Schulen aus dem als abschließendes Highlight vorgesehenen persönlichen Besuch einer kleineren Gruppe von indischen Schülerinnen und Schülern in Österreich mitnehmen?

Wenn sich die Jugendlichen aus beiden Ländern wirklich offen an der geplanten Zusammenarbeit beteiligen und es zu einem gegenseitigen inhaltlichen Austausch auf Augenhöhe kommt, könnte ihnen das Projekt „AustrIndia-4QOL“ eine große persönliche Bereicherung bieten. Damit wird versucht, sie nicht nur in ihrer Bereitschaft zu stärken, sich mit Jugendlichen aus einem anderen Kulturkreis vorurteilsfreier auseinander zu setzen, sondern darüber hinaus zu lernen, mit ihnen gleichberechtigt zusammenzuarbeiten. Über die Auseinandersetzung mit neuen und für sie eventuell fremdartigen Werthaltungen werden sich 
die Jugendlichen möglicherweise auch ihrer eigenen Einstellungen und Werte verstärkt bewusst.

\section{$4 \quad$ Fazit}

Mit dem Projekt „AustrIndia-4QOL“ wird versucht, die Auswirkungen einer Zusammenarbeit über digitale Medien von indischen und österreichischen Jugendlichen auf deren Einstellungen zu Fragen der Nachhaltigkeit zu analysieren. Dabei wird der Aspekt der intragenerationalen Gerechtigkeit in den Fokus gestellt. Zudem wird durch die Zusammenarbeit eine Verringerung gegenseitiger Stereotype angestrebt. Im Sinne einer moderat-konstruktivistischen Herangehensweise entscheiden sich die Schüler/innen gemeinsam für Forschungsthemen, entwickeln in Kleingruppen eigene Forschungsfragen, suchen nach entsprechenden Antworten und diskutieren ihre Ergebnisse mit den Jugendlichen des jeweils anderen Landes. Damit werden sie direkt in den Forschungsprozess eingebunden. Zudem wird mit diesem Lernsetting versucht, den Austausch zwischen Jugendlichen aus Österreich und Indien tatsächlich auf Augenhöhe durchzuführen. Ein kognitiver Wissenszuwachs durch die Beschäftigung in Kleingruppen mit den verschiedenen Themen ist erwünscht, aber nicht das vordergründige Ziel des Projekts „AustrIndia-4QOL“ und daher nicht Teil der wissenschaftlichen Analyse. Vielmehr wird primär das Ziel verfolgt, Jugendliche in ihrer Kompetenz zu stärken, globale Herausforderungen aus einer multiperspektivischen Sichtweise beurteilen zu können und verstärkt Aspekte der intragenerationalen Gerechtigkeit in ihre Überlegungen und Handlungen mit einzubeziehen. Die Fähigkeit zur interkulturellen Kooperation wird in zahlreichen $\mathrm{Pu}$ blikationen als Schlüsselkompetenz im Umgang mit globalen Herausforderungen bezeichnet (z. B.: Nagel 2010; Schmidtke 2009; Schreiber 2010; Schuler \& Kanwischer 2013). Nur durch gemeinsame Anstrengungen über Grenzen hinweg, können Lösungsansätze für globale Herausforderungen umgesetzt werden. Dies ist eine unabdingbare Voraussetzung, um im Sinne einer partizipativen Vorgehensweise die Bedingungen für hohe Lebensqualität für alle Menschen auf unserem Planeten zu schaffen.

\section{Literatur}

Applis, S. (2014): Die soziale Dimension des kommunikativen Aushandelns von Gerechtigkeitsfragen. Zur Bedeutung diskursiver unterrichtlicher Auseinandersetzungen in komplexen sozialen Handlungsfeldern. In: Geographie aktuell \& Schule 208 (36), 15-23.
Asbrand, B. (2008): Wie erwerben Jugendliche Wissen und Handlungsorientierung in der Weltgesellschaft? Globales Lernen aus der Perspektive qualitativ-rekonstruktiver Forschung. In: Zeitschrift für internationale Bildungsforschung und Entwicklungspädagogik (ZEP) 31 (1), 4-8.

Asbrand, B. (2009): Wissen und Handeln in der Weltgesellschaft. Eine qualitativ-rekonstruktive Studie zum Globalen Lernen in der Schule und in der außerschulischen Jugendarbeit. In: Asbrand, B., H. Hartmeyer \& G. Lang-Wojtasik (Hrsg.): Erziehungswissenschaft und Weltgesellschaft, 1. Münster: Waxmann.

BMBF - Bundesministerium für Bildung und Frauen (Hrsg.) (2015): Das Unterrichtsprinzip „Interkulturelles Lernen“. Wien. https://www.bmbf.gv.at/schulen/ unterricht/prinz/interkult_lernen.html (18.08.2015).

BMUKK - Bundesministerium für Unterricht, Kunst und Kultur, BMLFUW - Bundesministerium für Land- und Forstwirtschaft, Umwelt und Wasserwirtschaft \& BMWF - Bundesministerium für Wissenschaft und Forschung (Hrsg.) (2008): Österreichische Strategie zur Bildung für nachhaltige Entwicklung. Wien.

Budke, A. (2013): Interkulturelles Lernen im Geographieunterricht. In: Kanwischer, D. (Hrsg.): Geographiedidaktik. Ein Arbeitsbuch zur Gestaltung des Geographieunterrichts. Studienbücher der Geographie. Stuttgart: Borntraeger.

De Haan, G. (2008): Gestaltungskompetenz als Kompetenzkonzept der Bildung für nachhaltige Entwicklung. In: Bormann, I., G. de Haan (Hrsg.): Kompetenzen der Bildung für nachhaltige Entwicklung. Operationalisierung, Messung, Rahmenbedingungen, Befunde. Wiesbaden: Verlag für Sozialwissenschaften, 23-44.

Government of India, Ministry of Home Affairs (2011): Census of India. http://www.censusindia.gov.in/pca/ default.aspx (20.01.2015).

Hahn, H. (2009): Globale Gerechtigkeit. Eine philosophische Einführung. Frankfurt: Campus Verlag.

Hauff, M. (2012): Nachhaltige Entwicklung - Begründung und Anforderungen des neuen Leitbildes. Bonn: Online Akademie. http://www.fes-online-akademie. de/fileadmin/Inhalte/01_Themen/03_Nachhaltigkeit/ dokumente/FES_OA_Leitbild_nachhalt._Entwicklung. $\operatorname{pdf}(30.07 .2015)$.

Heintel, P. (2007): Kulturelle Nachhaltigkeit. Eine Annäherung. In: Krainer, L. \& R. Trattnigg (Hrsg.): Kulturelle Nachhaltigkeit. Konzepte, Perspektiven, Positionen. Oekom: München, 65-168.

Helm, F. (2013): A Dialogic Model for Telecollaboration. In: Bellaterra Journal of Teaching \& Learning Language \& Literature 6 (2), 28-48.

Höffe, O. (2015): Gerechtigkeit. In: Krell, C. \& T. Mörschel (Hrsg.): Werte und Politik. Wiesbaden: Springer VS, 37-50.

Hofmann, R. \& E.M. Ulrich-Riedhammer (2014): Die Konstruktion (inter-)kultureller Räume als moralischethische Urteile. Entfaltung ethischer Urteilskompetenz als kritisch-reflexiver Umgang mit Raumkonstruktionen im Geographieunterricht. In: Schrüfer G. \& I. Schwarz 
(Hrsg.): Globales Lernen. Ein geographischer Diskursbeitrag. Münster: Waxmann, 155-170.

Höhnle, S. (2014): Online-gestützte Projekte im Kontext Globalen Lernens im Geographieunterricht. Empirische Rekonstruktion internationaler Schülerperspektiven. In: Hemmer, M., J. Nebel \& Y. Schleicher (Hrsg.): Geographiedidaktische Forschungen 53. Münster.

Interkulturelles Zentrum (Hrsg.) (2014): Nachhaltigkeit macht Schule! Empfehlungen für erfolgreiche und qualitative Schulpartnerschaften weltweit. Wien.

Jones, C. W. (2014): Exploring the Microfoundations of International Community: Toward a Theory of Enlightened Nationalism. In: International Studies Quarterly 58, 682-705.

Kammerl, R. (2009): Web 2.0. Neue Medien - neue Chancen für Partizipation und Demokratieerziehung? In: Zeitschrift für internationale Bildungsforschung und Entwicklungspädagogik 32 (3), 9-13.

Keller, L. \& A. Oberrauch (2013): Lebensqualitätsforschung mit Jugendlichen vor dem Hintergrund eines neuen österreichischen Kompetenzmodells. In: Institut für Geographie \& Innsbrucker Geographische Gesellschaft (Hrsg.): Innsbrucker Jahresbericht 2011-2013. Innsbruck, 103-120.

Keller, L. \& A. Schober (2011): „LIFE eQuality?” - Beginn eines Projekts zur Untersuchung der Lebensqualität von Jugendlichen durch Jugendliche in vier Alpenregionen. In: Innsbrucker Geographische Gesellschaft (Hrsg.): Innsbrucker Jahresbericht 2008-2010. Innsbruck, 188201.

Krainer, L. \& R. Trattnigg (2007): Nachhaltigkeit ist eine Frage der Kultur. In: Krainer, L. \& R. Trattnigg (Hrsg.): Kulturelle Nachhaltigkeit. Konzepte, Perspektiven, Positionen. Oekom: München, 6-28.

Kuckartz, U. (2014a): Mixed Methods. Methodologie, Forschungsdesgins und Analyseverfahren. Wiesbaden: Springer VS.

Kuckartz, U. (2014b): Qualitative Inhaltsanalyse. Methoden, Praxis, Computerunterstützung. 2. Aufl., Weinheim: Beltz Juventa.

Kyburz-Graber, R. (2010): Nachhaltige Entwicklung im Unterricht umsetzen: Rahmenkonzept und Unterrichtsformen. In: Kyburz-Graber, R., U. Nagel \& F. Odermatt (Hrsg.): Handeln statt hoffen. Materialien zur Bildung für Nachhaltige Entwicklung für die Sekundarstufe I. Zug: Klett und Balmer, 15-27.

Lamy, M.-N. \& R. Goodfellow (2010): Telecollaboration and Learning 2.0. In: Guth, S. \& F. Helm (eds.): Telecollaboration 2.0. Language, Literacies and Intercultural Learning in the $21^{\text {th }}$ Century. Bern, 107-138.

Lang-Wojtasik, G. \& A. Scheunpflug (2002): Bildung durch Begegnungsreisen? Interkulturelles Lernen in Zeiten des Massentourismus. In: Kreienbaum, M.-A. et al. (Hrsg.):Bildung als Herausforderung-Leben und Lernen in Zambia. Frankfurt am Main, London, 17-35.

Lang-Wojtasik, G. \& A. Scheunpflug (2005): Kompetenzen Globalen Lernens. In: Zeitschrift für internationale Bildungsforschung und Entwicklungspädagogik (ZEP) 28 (2), 2-7.
Leitner, M., A. Christanell, C. Bertsch \& K-M. Brunner (2011): my life - my style - my future. Nachhaltige Lebensstile und jugendliche Lebenswirklichkeiten. Endbericht. Wien: ÖIN - Österreichisches Institut für nachhaltige Entwicklung.

McCarthy, J. (2012): International design collaboration and mentoring for tertiary students through Facebook. In: Australasian Journal of Educational Technology 28 (5), 755-775. http://ajet.org.au/index.php/AJET/ article/view/1383/602 (21.11.2015).

Nagel, U. (2010): Nachhaltige Entwicklung verstehen: die Hintergründe. In: Kyburz-Graber, R., U. Nagel \& F. Odermatt (Hrsg): Handeln statt hoffen. Materialien zur Bildung für Nachhaltige Entwicklung für die Sekundarstufe I. Zug: Klett und Balmer, 10-14.

Nohl, A-M.(2010): Konzepte interkultureller Pädagogik. Eine systematische Einführung. 2. Aufl., Bad Heilbrunn: Klinkhardt.

Oberrauch, A., L. Keller, P. Sanin \& M. Riede (2014): Lebensqualitätsvorstellungen von Jugendlichen im Kontext des Leitbilds nachhaltiger Entwicklung. In: Mitteilungen der österreichischen geographischen Gesellschaft 156, 221-248.

Oberrauch, A. \& L. Keller (2015): Methodenkombination in der Conceptual Change-Forschung. Komplexität in multiperspektivischen Forschungsdesigns gerecht werden. In: Budke, A. \& M. Kuckuck (Hrsg.): Geographiedidaktische Forschungsmethoden. (= Praxis Neue Kulturgeographie). Münster: LIT-Verlag, 87-109.

Oberrauch, A. \& L. Keller (in Vorbereitung): SchülerInnen-Vorstellungen zur Lebensqualität und ihre Veränderung in moderat konstruktivistischen Lernsettings - ein Beitrag zur Bildung für nachhaltige Entwicklung.

Obrecht, A. (2014): Wozu wissen wollen? Wissen - Herrschaft - Welterfahrung. Wien: Edition Ausblick.

OECD - Organisation für wirtschaftliche Zusammenarbeit und Entwicklung (Hrsg.) (2005): Definition und Auswahl von Schlüsselkompetenzen. Zusammenfassung. Paris. http://www.oecd.org/pisa/35693281.pdf (17.09.2015).

OECD - Organisation für wirtschaftliche Zusammenarbeit und Entwicklung (Hrsg.) (o. J.): Better Life Index. Was ist den Menschen weltweit am wichtigsten? Paris. http:// www.oecdbetterlifeindex.org/de/countries/austria-de/ (20.09.2015).

Oehme, I. (2007): Stand der Nachhaltigkeitsdiskussion: Eine Übersicht zur Konzeptualisierung im deutschsprachigen Raum. In: Krainer, L. \& R. Trattnigg (Hrsg.): Kulturelle Nachhaltigkeit. Konzepte, Perspektiven, Positionen. Oekom: München, 203-222.

Reinfried, S. (2007): Alltagsvorstellungen und Lernen im Fach Geographie. Zur Bedeutung der konstruktivistischen Lehr-Lern-Theorie am Beispiel des Conceptual Change. In: Geographie und Schule 168, 19-28.

Reinfried, S., B. Rottermann, U. Aeschbacher \& E. Huber (2010): Alltagsvorstellungen über den Treibhauseffekt und die globale Erwärmung verändern - eine Voraussetzung für Bildung für nachhaltige Entwicklung. In: Schweizerische Zeitschrift für Bildungswissenschaften $32,251-273$. 
Reinfried, S. (2015): Wissen erwerben und Einstellungen Reflektieren. In: Reinfried, S. \& H. Haubrich (Hrsg.): Geographie unterrichten lernen. Die Didaktik der Geographie. Berlin: Cornelsen, 53-98.

Rhode-Jüchtern, T. (2009): Eckpunkte einer modernen Geographiedidaktik. Hintergrundbegriffe und Denkfiguren. Seelze-Velber: Klett und Kallmeyer.

Rhode-Jüchtern, T. (2011): Gestaltung von Lernumgebungen im Geographieunterricht. In: Kanwischer, D. (Hrsg.): Geographiedidaktik. Ein Arbeitsbuch zur Gestaltung des Geographieunterrichts. Studienbücher der Geographie. Stuttgart: Borntraeger, 105-116.

Scheunpflug, A. (2008): Die konzeptionelle Weiterentwicklung des Globalen Lernens. Die Debatten der letzten zehn Jahre. In: VENRO (Hrsg.): Jahrbuch Globales Lernen 2007/2008: Standortbestimmung - Praxisbeispiele - Perspektiven. Bielefeld: Welthaus, 11-21.

Scheunpflug, A. \& N. Schröck (2002): Globales Lernen. Einführung in eine pädagogische Konzeption zur entwicklungsbezogenen Bildung. Stuttgart.

Schmidt-Wulffen, W. (2001): Bilder über „Fremde“. Wie deutsche und ghanaische Schüler einander und sich selbst sehen. In: Geographie heute 190, 13-15.

Schmidt-Wulffen, W. (2008): Konstruktivismus im GWUnterricht - oder: „Wir wollen dir nicht die Welt erklären, sondern helfen, diese für dich zu entdecken“. In: Dobler, K., T., Jekel \& H. Pichler (Hrsg.): kind:macht:raum. Wichmann: Heidelberg, 76-86.

Schleicher, Y. (2006): Digitale Medien und E-Learning - ein Beitrag zum Globalen Lernen im Geographieunterricht? In: Zeitschrift für internationale Bildungsforschung und Entwicklungspädagogik 29 (3), 13-17.

Schmidtke, V. (2009): Glossar: Hintergrundbegriffe für eine moderne Geographiedidaktik. In: Rhode-Jüchtern, T. (Hrsg.): Eckpunkte einer modernen Geographiedidaktik. Hintergrundbegriffe und Denkfiguren. Seelze-Velber: Klett und Kallmeyer, 143-189.

Schreiber, J.R. (2010): Das Globale Lernen in der Dekade „Bildung für nachhaltige Entwicklung“ - Halbzeitbilanz und Perspektiven. In: VENRO (Hrsg.): Jahrbuch Globales Lernen 2010. Globales Lernen als Herausforderung für Schule und Zivilgesellschaft. Bielefeld: Welthaus, 35-42.

Schreiber, J.R., K. Edler \& M. Schawe (2010): Didaktisches Konzept. In: Globales Lernen. Hamburger Unterrichtsmodelle zum KMK-Orientierungsrahmen Globale Entwicklung, 1. Hamburg.

Schrüfer, G. (2004): Ich wünschte, ich könnte die Welt durch deine Augen sehen - und du durch meine. In: Praxis Geographie 34 (1), 46-47.

Schrüfer, G. (2010): Förderung interkultureller Kompetenz im Geographieunterricht. Ein Beitrag zum Globalen Lernen. In: Schrüfer G. \& I. Schwarz (Hrsg.): Globales Lernen. Ein geographischer Diskursbeitrag. Münster: Waxmann, 101-110.

Schrüfer, G. \& I. Obermaier (2014): Wahrnehmung von Räumen. Konstruktion und Bewertung aus unter- schiedlichen Perspektiven. In: Schwarz, I. \& G. Schrüfer (Hrsg.): Vielfältige Geographien. Entwicklungslinien für Globales Lernen, Interkulturelles Lernen und Wertediskurse. Münster: Waxmann, 171-186.

Schuler, S. (2013): Geographielernende - Interesse und Vorwissen. In: Kanwischer, D. (Hrsg.): Geographiedidaktik. Ein Arbeitsbuch zur Gestaltung des Geographieunterrichts. Studienbücher der Geographie. Stuttgart: Borntraeger, 81-91.

Schuler, S. \& D. Kanwischer (2013): Bildung für nachhaltige Entwicklung: Globales Lernen und Umweltbildung im Geographieunterricht. In: Kanwischer, D. (Hrsg.): Geographiedidaktik. Ein Arbeitsbuch zur Gestaltung des Geographieunterrichts. Studienbücher der Geographie. Stuttgart: Borntraeger, 164-175.

Sedmak, C. (Hrsg.) (2014): Gerechtigkeit. Vom Wert der Verhältnismäßigkeit. Darmstadt: WBG.

Thomas, A., C. Chang \& H. Abt (2007): Erlebnisse die verändern. Langzeitwirkungen der Teilnahme an internationalen Jugendbegegnungen. Göttingen.

Thomas, A. \& D. Perl (2010): Chancen, Grenzen und Konsequenzen interkulturellen Lernens im internationalen Schüleraustausch. In: Fachstelle für Internationale Jugendarbeit der Bundesrepublik Deutschland e.V. (Hrsg.): Forum Jugendarbeit International. Bonn, 286-302.

UNESCO - Organisation der Vereinten Nationen für Erziehung, Wissenschaft und Kultur (Hrsg.) (2014b): Roadmap zur Umsetzung des Weltaktionsprogramms „Bildung für nachhaltige Entwicklung“. Bonn. http:// www.bne-portal.de/fileadmin/unesco/de/Downloads/ Dekade_Publikationen_international/2015_Roadmap_ deutsch.pdf (26.08.2015).

UNESCO - Organisation der Vereinten Nationen für Erziehung, Wissenschaft und Kultur (eds.) (2015a): Education for Sustainable Development. Paris. http:// www.unesco.org/new/en/education/themes/leadingthe-international-agenda/education-for-sustainabledevelopment/ (18.08.2015).

UNESCO - Organisation der Vereinten Nationen für Erziehung, Wissenschaft und Kultur (eds.) (2015b): Rethinking Education. Towards a global common good? Paris. http://unesdoc.unesco.org/ images/0023/002325/232555e.pdf (18.08.2015).

Wang, Q. H.L. Woo, C.L. Quek, Y. Yang \& M. Liu (2012): Using the Facebook group as a learning management system: An exploratory study. In: British Journal of Educational Technology 43 (3), 428-438.

WCED - World Commission on Environment and Development (1987): Our Common Future, Oxford University Press, Oxford.

WHO - Weltgesundheitsorganisation \& UNICEF Kinderhilfswerk der Vereinten Nationen (eds.) (2014): Progress on sanitation and drinking-water - 2014 update. Genf: WHO Press. http://apps.who.int/iris/ bitstream/10665/112727/1/9789241507240_eng.pdf (18.08.2015). 